Tohoku J. Exp. Med., 2004, 204, 63-69

\title{
Assessment of Cardiac Autonomic Regulation in Children with Monosymptomatic Nocturnal Enuresis by Analysis of Heart Rate Variability
}

\author{
Murat Unalacak, Mustafa Aydin, ${ }^{1}$ Bahri Ermis, ${ }^{2}$ Ali Ozeren, ${ }^{1}$ Ayhan Sogut, ${ }^{2}$ \\ FAtma Demirel $^{2}$ and Ilhami Unluoglu ${ }^{3}$ \\ Departments of Family Medicine, ${ }^{1}$ Cardiology and ${ }^{2}$ Pediatrics, Faculty of \\ Medicine, Zonguldak Karaelmas University, Zonguldak, Turkey, and \\ ${ }^{3}$ Department of Family Medicine, Osmangazi University, Eskisehir, Turkey
}

Unalacak, M., Aydin, M., Ermis, B., Ozeren, A., Sogut, A., Demirel, F. and Unluoglu, I. Assessment of Cardiac Autonomic Regulation in Children with Monosymptomatic Nocturnal Enuresis by Analysis of Heart Rate Variability. Tohoku J. Exp. Med., 2004, 204 (1), 63-69 — The pathogenesis of monosymptomatic nocturnal enuresis is controversial. Various urodynamic studies showed bladder hyperactivity in enuretic children. But the exact cause is not precisely known. The aim of this study was to understand whether the autonomic nervous system dysfunction is involved in this bladder hyperactivity or not. Heart rate variability measurement is widely used for evaluation of cardiac autonomic activity. We evaluated cardiac autonomic nervous system functions in monosymptomatic nocturnal enuretic children by using 24-hour Holter electrocardiogram. The study group consisted of 32 enuretic children (20 boys and 12 girls) and the control group consisted of 20 healthy children (12 boys and 8 girls). In these two growps, we assessed cardiac autonomic regulation by analysis of heart rate variability, and found a significantly higher parasympathetic activity in enuretic children than controls. We suggest that the parasympathetic nervous system hyperactivity plays a role in nocturnal enuresis by causing vesical hyperactivity in monosymptomatic enuretic children. - monosymptomatic nocturnal enuresis; autonomic nervous regulation; heart rate variability; children (C) 2004 Tohoku University Medical Press

Received May 17, 2004; revision accepted for publication July 6, 2004.

Address for reprints: Dr. Murat Unalacak, Department of Family Medicine, Faculty of Medicine, Zonguldak Karaelmas University, Zonguldak, Turkey.

e-mail:drunalacak@yahoo.com 
Enuresis is defined as involuntary voiding; when it occurs at night, it is termed nocturnal enuresis, and daytime incontinence is termed diurnal enuresis. More than $80 \%$ of enuretics wet only at night and have no other abnormalities in their urologic history; they are classified as having monosymptomatic nocturnal enuresis (Scoot and Dalton 2000; Bauer et al. 2002).

The autonomic nervous system link through which brain commands the heart. This is performed through sympathetic and parasympathetic fibers innervating sinus node, and by this way, heart rate and rhythm are altered. ECG is a suitable choice for assesment of sinus rhythm for evaluation of autonomic outflow on heart. The time domain and the frequency domain approaches use non-invasive recordings and makes it possible to evaluate autonomic modulation of cardiovascular function (Pagani et al. 1986; Akselrod 1988; Malliani et al. 1991).

Basic heart rate and its modulation are primarily due to alterations in autonomic tone; parasympathetic tone slowing the heart rate and sympathetic stimulation increasing the heart rate. These heart rate fluctuations can be evaluated quantitatively by ambulatory electrocardiogram recordings and provide an evaluation of autonomic functions (Akinci et al. 1993). Heart rate variability (HRV) is a useful tool that might provide indices of autonomic modulation of the sinus node (Task Force of the European Society of Cardiology and the North American Society of Pacing and Electrophysiology 1996). Changes in heart rate and HRV can be measured by various techniques and since these changes are autonomically mediated, these measurements may reflect autonomic tone. By the power spectral analysis of HRV, the high frequency component (HF) demonstrates parasympathetic activity and the low frequency component (LF) shows mainly sympathetic activity, although the LF reflects partial parasympathetic activity too. The LF/HF ratio shows sympathetic activity (Finley and Nugent 1995; Kautzner and Hnatkova 1995). Urodynamic studies have shown the presence of bladder hyperactivity in a number of enuretics (Booth and Gosling 1983; Wu et al. 1988; Butler 1994). The cause of hyperactivity is not clear, but the autonomic nervous system dysfunction may be involved (Yakinci et al. 1997). The previous studies, which used time domain variables, showed that enuretics have sympathetic activity (Dundaroz et al. 2001). In another study, it was showed by using power spectral analysis that enuretics have parasympathetic activity (Fujiwara et al. 2001). Due to these conflicting results, we planned in this study to investigate autonomic nervous system function in monosymtomatic enuretic children using 24-hour ambulatory electrocardiography, both by time dependent and power spectral HRV analysis.

\section{Materials ANd Methods}

The study group consisted of 32 children with monosymptomatic nocturnal enuresis (MNE); 20 boys and 12 girls, and the control group consisted of 20 healthy age-sex matched children; 12 boys and 8 girls. The mean ages were $8.0 \pm 1.6$ years (range 5-12) and $8.0 \pm 1.9$ years (range 6-13), respectively. We diagnosed a patient as enuresis when urine is voided twice a week for at least 3 consecutive months or clinically significant distress occurs in areas of the child's life as a result of the wetting (Scoot and Dalton 2000).

To exclude urologic or neurologic abnormalities, a detailed case history was taken and a physical examination was performed. In addition, routine blood chemistry, urine analysis, urine culture and urinary ultrasound were carried out in all of the children in order to eliminate deficiency of anti-diuretic hormone, urinary tract infection, sickle cell disease/trait, diabetes mellitus and diabetes insipidus. All tests were within normal limits in both study group and control group. No child in either group had autonomic symptoms such as fatigue, syncope, etc. None of the patients has been using any medication at the time of study. After taking written consent, 24-hour Holter electrocardiogram test was carried out on 
all children in both groups.

This study has been approved by the ethical committee of our institute.

\section{Holter monitoring}

All patients and control subjects underwent 24-hour Holter recording with 3-channel real-time flash card; a digital recorder (Brentwood by Midmark 8800 Holter Torrance, CA, USA), monitoring the bipolar leads of ECG V2, V5, and modified aVF. Holter flash cards were analyzed with the software version 5.2 Brentwood HRV analysis package.

\section{HRV analysis}

Both time domain and frequency domain (power spectral) analyses were used in this study to assess HRV data. Time domain analysis of Holter recordings employs a variety of measures that provide a statistical analysis of the fluctuations in sinus R-R intervals over 24 hours (Kleiger et al. 1995). Collectively, time domain measures quantify how much variability exists in heart rate over a 24-hour period. The time domain HRV measures employed in this study were mean heart rate, the standard deviation of all normal sinus R-R intervals over 24 hours (SDNN), the standard deviation of all averaged normal sinus R-R intervals for each 5-minute segment in the 24-hour recording (SDANN), and the root mean square of successive differences between normal sinus R-R intervals (rMSSD). Frequency analysis of HRV using Fast Fourier transformation (a special method used for evaluation of heart rate variability) reveals peaks at several major frequency ranges, which have been defined as the high frequency (HF) $(0.15-<0.4 \mathrm{~Hz})$, low frequency (LF) $(0.04-<0.15 \mathrm{~Hz})$, and very low frequency (VLF) $(0.0033-<0.04 \mathrm{~Hz})$ regions of the power spectrum (Finley and Nugent 1995; Task Force of the European Society of Cardiology and the North American Society of Pacing and Electrophysiology 1996). Holter exclusion criteria were: a duration of Holter monitoring shorter than 20 hours; the presence of significant artefacts; more than 200 isolated premature atrial or ventricular beats or any repetitive form; sinus pauses longer than 2 s (Scott et al. 1980), any degree of atrioventricular block, even if present only during sleep time. In this regard, subjects with first degree atrio-ventricular block were excluded because it is found as either a normal variant or in different congenital or acquired heart diseases, systemic diseases, metabolic abnormalities (Friedman 1998). Subjects with second degree Mobitz type 1 atrio-ventricular block were excluded because in the presence of blocked $P$ waves, R-R intervals (where HRV is calculated) differ from P-P intervals, and give rise to a false nonsinus longer R-R interval (Kamath and Fallen 1995). The presence of sporadic atrial ectopic rhythm (such as coronary sinus rhythm) was not a criterion of exclusion (Scott et al. 1980).

\section{Statistical analysis}

Results were expressed as mean \pm S.D. In order to check the normality assumption the Kolmogorov-Smirnov test was applied. MannWhitney's U-test was applied in order to compare mean values of enuretic group and healthy children. A $p$-value $<0.05$ was considered statistically significant. Data analyses were carried out using SPSS 9.0 statistics programme (SPSS Inc., Chicago, IL, USA).

\section{Results}

The cardiac autonomic activity function by the time dependent analysis findings of heart rate variability using 24-hour Holter electrocardiograms are listed in Table 1.

SDNN and SDANN increased significantly in MNE children compared to healthy children $(p<0.01, p<0.01$ respectively). rMSSD also increased significantly in MNE compared to healthy children $(p<0.01)$.

The cardiac autonomic activity function by the power spectrum analysis findings of heart rate variability using 24-hour Holter electrocardiograms are listed in Table 2. VLF and LF decreased significantly in MNE compared to healthy 
TABLE 1. The time dependent analysis of heart rate variability (HRV) using 24-hour Holter electrocardiogram

\begin{tabular}{lccc}
\hline & MNE $(n=32)$ & Control $(n=20)$ & $p$ \\
\hline SDNN & $140 \pm 30$ & $120 \pm 28$ & $<0.01$ \\
SDANN & $127 \pm 36$ & $106 \pm 37$ & $<0.01$ \\
RMSSD & $44 \pm 13$ & $33 \pm 9$ & $<0.01$ \\
\hline
\end{tabular}

SDNN, the standard deviation of all normal sinus R-R intervals over 24 hours; SDANN, standard deviation of all averaged normal sinus intervals for each 5-minute segment in the 24-hour recording; rMSSD, the root mean square of successive differences between normal sinus R-R intervals.

TABLE 2. The power spectrum analysis of heart rate variability (HRV) using 24-hour Holter electrocardiogram

\begin{tabular}{lccc}
\hline & MNE $(n=32)$ & Control $(n=20)$ & $p$ \\
\hline Total power & 1774 & 1720 & NS \\
VLF $\left(\mathrm{ms}^{2}\right)$ & $564 \pm 371$ & $627 \pm 387$ & $<0.05$ \\
LF $\left(\mathrm{ms}^{2}\right)$ & $202 \pm 102$ & $266 \pm 110$ & $<0.05$ \\
$\mathrm{HF}\left(\mathrm{ms}^{2}\right)$ & $239 \pm 125$ & $160 \pm 109$ & $<0.01$ \\
$\mathrm{LF} / \mathrm{HF}\left(\mathrm{ms}^{2}\right)$ & $1.02 \pm 0.46$ & $1.44 \pm 0.36$ & $<0.01$ \\
\hline
\end{tabular}

VLF, very low frequency component; LF, low frequency component; HF, high frequency component; LF/HF, the ratio of low frequency component and high frequency component; NS, non-significant.

children $(p<0.05, p<0.05$ respectively). HF increased significantly in MNE compared to healthy children $(p<0.01)$, and LF/HF significantly decreased in MNE compared to healthy children $(p<0.01)$. There was no significant difference of HRV between males and females.

\section{Discussion}

It has been suggested that one of the reasons of MNE in children is autonomic dysfunction. There are several investigations using only one method to evaluate MNE. Fujiwara et al. (2001) demonstrated the dominance of parasympathetic activity by using only power spectral analysis method. On the other hand, Dundaroz et al. (2001) demonstrated the dominance of sympathetic activity by using only time dependent analysis method. In our study, we used both of these two methods to evaluate MNE.

According to the urodynamic studies, blad- der instability and hyperactivity seems as an important etiological factor in the MNE pathogenesis (Wu et al. 1988; Yakinci et al. 1997; Cigna et al. 2002). According to our study parasympathetic hyperactivity could be a responsible factor for bladder hyperactivity. In another study, it has been supposed that etiopathogenesis of MNE could be multifactorial (Butler 1994). Thus, our results give us a partial explanation to understand some parts of etiopathogenesis of MNE.

The present study has shown that parasympathetic nervous system functions are hyperactive in children with MNE. There are many studies, which evaluated the cardiac autonomic regulation by the power spectral analysis and time dependent analysis using 24-hour Holter electrocardiogram (Goto et al. 1997; Massin et al. 1997; Makimattila et al. 2000; Martini et al. 2001; Yang et al. 2001; Kazuma et al. 2002; Mehta et al. 2002; Park et al. 2002). The power spectrum of heart rate variabil- 
ity was analyzed in three frequency components consisting of the very low frequency component and high frequency component. The HF, which is related to respiratory sinus arrhythmia, is widely accepted as a measure of parasympathetic or vagal activity (Task Force of the European Society of Cardiology and the North American Society of Pacing and Electrophysiology 1996). We found significantly higher HF results in MNE group than control group. Power in the LF band is related to baroreceptor activity and blood pressure wave activity and resonance, and is dually modulated by the parasympathetic and sympathetic systems (Pomeranz et al. 1985; Malliani 1999). In our study, LF values were significantly lower than control group. The ratio of $\mathrm{LF} / \mathrm{HF}$ power has been used as a marker of sympathovagal balance in some studies (Malliani 1999), however, because LF power can reflect both sympathetic and parasympathetic activity, this interpretation can at times be misleading (Eckberg 1997).

Although the majority of the total power in the 24-hour HRV power spectrum is accounted for by VLF components, the physiological mechanisms involved in the generation of these rhythms are less defined and still somewhat controversial. The VLF component has been proposed as a marker of sympathetic activity (Perini et al. 2000). In our study, VLF band was significantly decreased.

For time-domain analysis, several statistical variables are derived from a recorded heart rate signal. Despite their simplicity, these measurements, the most common of which is standard deviation (square root of variance), proved to be useful clinical tools (Task Force of the European Society of Cardiology and the North American Society of Pacing and Electrophysiology 1996). The standard deviation provides an evaluation of overall HRV, however, as a measure of changes in sympathovagal balance, it is limited. This standard deviation is also referred to as the SDNN. The most commonly used measure derived from interval differences is the RMSSD, which is an estimate of HF variation in heart rate.
Frequency-domain methods provide the basic information of how power (variance) distributes as a function of frequency (Task Force of the European Society of Cardiology and the North American Society of Pacing and Electrophysiology 1996).

HRV is usually evaluated by frequency and time-domain analysis (Kleiger et al. 1995; Task Force of the European Society of Cardiology and the North American Society of Pacing and Electrophysiology 1996) with short and long-term recordings. In some conditions, long-term (24-hour) recordings have been more sensitive in detecting abnormalities in autonomic function than short-term variables (Kleiger et al. 1995; Task Force of the European Society of Cardiology and the North American Society of Pacing and Electrophysiology 1996). Therefore, we recorded and analyzed all findings for 24 hours in MNE positive children in order to detect variabilities in autonomic activities. To our knowledge, there is no study using both investigational methods together in the literature.

In our study, we found the dominance of parasympathetic activity using spectral and time domain analysis methods. In time domain analysis, there were significant decreases for SDNN and SDANN values in MNE group. In spectral analysis, there were significantly increased HF and decreased LF values in the MNE group. These results confirm the parasymphatetic dominance in the etiopathogenesis of MNE. Besides, anticholinergic treatment may have beneficial effects for these patients.

\section{Conclusion}

We detected parasympathetic nervous system hyperactivity in MNE children through the 24-hour analysis. This indicates that vesical hyperactivity can be caused by parasympathetic nervous system hyperactivity in enuretic children. 


\section{References}

Akinci, A., Celiker, A., Baykal, E. \& Tezic, T. (1993) Heart rate variability in diabetic children: sensitivity of the time- and frequency-domain methods. Pediatr. Cardiol., 14, 140-146.

Akselrod, S. (1988) Spectral analysis of fluctuations in cardiovascular parameters: a quantitative tool for the investigation of autonomic control. Trends Pharmacol. Sci., 9, 6-9.

Bauer, S.B., Koff, S.A. \& Jayanthi, V.R. (2002) Neuropathic dysfunction of the lower urinary tarct. In: Campbell's Urology, edited by P.C. Walsh, A.B. Retik, E.D. Vaughan, A.J. Wein, Philadelphia, Saunders, pp. 2231-2283.

Booth, C.M. \& Gosling, J.A. (1983) Histology and urodynamic study of the bladder in enuretic children. Br. J. Urol., 55, 367-370.

Butler, R.J., editor (1994) Nocturnal Enuresis: The child's experience. Oxford; Butterworth Heinemann.

Cigna, R.M., Chiaramonte, C., Piscopo, A. \& Cataliotti, F. (2002) Urodynamic evaluation in children with enuresis. Pediatr. Med. Chir., 24, 363-367.

Dundaroz, M.R., Denli, M., Uzun, M., Aydin, H.I., Sarici, S.U., Yokusoglu, M. \& Ulgen, S. (2001) Analysis of heart rate variability in children with primary nocturnal enuresis. Int. Urol. Nephrol., 32, 393-397.

Eckberg, D.L. (1997) Sympathovagal balance: a critical appraisal. Circulation, 96, 3224 -3232.

Finley, J.P. \& Nugent, S.T. (1995) Heart rate variability in infants, children and young adults. J. Auton. Nerv. Syst., 51, 103-108.

Friedman, R.A. (1998) Sinus and atrioventricular conduction disorders. In: Current concept in diagnosis and management of arrhythmias in infants and children, edited by B. Deal, G. Wolff, H. Gelband, NY, Futura, pp. 89-116.

Fujiwara, J., Kimura, S., Tsukayama, H., Nakahara, S., Haibara, S., Fujita, M., Isobe, N. \& Tamura, K. (2001) Evaluation of the autonomic nervous system function in children with primary monosymptomatic nocturnal enuresis - power spectrum analysis of heart rate variability using 24-hour Holter electrocardiograms. Scand. J. Urol. Nephrol,. 35, 350-356.

Goto, M., Nagashima, M., Baba, R., Nagano, Y., Yokota, M., Nishibata, K. \& Tsuji, A. (1997) Analysis of heart rate variability demonstrates effects of development on vagal modulation of heart rate in healthy children. J. Pediatr., 130, 725-729.

Kamath, M.V. \& Fallen, E.L. (1995) Correction of the heart rate variability signal for ectopic and missing beats. In: Heart rate variability, edited by M. Malik, A.J. Camm, NY, Futura, pp. 75-85.

Kautzner, J. \& Hnatkova, K. (1995) Correspondence of different methods for heart rate variability measurement. In: Heart rate variability, edited by M. Malik, A.J. Camm, NY, Futura, pp. 119-126.

Kazuma, N., Otsuka, K., Wakamatsu, K., Shirase, E. \& Matsuoka, I. (2002) Heart rate variability in normotensive healthy children with aging. Clin. Exp. Hypertens., 24, 83-89.

Kleiger, R.E., Stein, P.K., Bosner, M.S. \& Rottman, J.N. (1995) Time-domain measurements of heart rate variability. In: Heart rate variability, edited by M. Malik, A.J. Camm, NY, Futura, pp. 33-45.

Makimattila, S., Schlenzka, A., Mantysaari, M., Bergholm, R., Summanen, P., Saar, P., Erkkila, H. \& Yki-Jarvinen, H. (2000) Predictors of abnormal cardiovascular autonomic function measured by frequence domain analysis of heart rate variability and conventional tests in patients with type 1 diabetes. Diabetes Care, 23, 1686-1693.

Malliani, A., Pagani, M., Lombardi, F. \& Cerutti, S. (1991) Cardiovascular neural regulation explored in the frequency domain. Circulation, 84, 482-492.

Malliani, A. (1999) The pattern of sympathovagal balance explored in the frequency domain. News Physiol. Sci., 14, 111-117.

Martini, G., Riva, P., Rabbia, F., Molini, V., Ferrero, G.B., Cerutti, F., Carra, R. \& Veglio, F. (2001) Heart rate variability in childhood obesity. Clin. Auton. Res., 11, 87-91.

Massin, M. \& von Bernuth, G. (1997) Normal ranges of heart rate variability during infancy and childhood. Pediatr. Cardiol., 18, 297-302.

Mehta, S.K., Super, D.M., Connuck, D., Salvator, A., Singer, L., Fradley, L.G., Harcar-Sevcik, R.A., Kirchner, H.L. \& Kaufman, E.S. (2002) Heart rate variability in healthy newborn infants. Am. J. Cardiol., 89, 50-53.

Pagani, M., Lombardi, F., Guzzetti, S., Rimoldi, O., Furlan, R., Pizzinelli, P., Sandrone, G., Malfatto, G., Dell'Orto, S., Piccaluga, E., Turiel, M., Baselli, G., Cerutti, S. \& Malliani, A. 
(1986) Power spectral analysis of heart rate and arterial pressure variabilities as a marker of sympatho-vagal interaction in man and conscious dog. Circ. Res., 59, 178-193.

Park, E.S., Park, C.I., Cho, S.R., Lee, J.W. \& Kim, E.J. (2002) Assessment of autonomic nervous system with analysis of heart rate variability in children with spastic cerebral palsy. Yonsei Med. J., 43, 65-72.

Perini, R., Fisher, N., Veicsteinas, A. \& Pendergast, D.R. (2000) Heart rate variability during dynamic exercise in elderly males and females. Eur. J. Appl. Physiol., 82, 8-15.

Pomeranz, B., Macaulay, R.J., Caudill, M.A., Kutz, I., Adam, D., Gordon, D., Kilborn, K.M., Barger, A.C., Shannon, D.C., Cohen, R.J. \& Benson, N. (1985) Assessment of autonomic function in humans by heart rate spectral analysis. Am. J. Physiol., 248, 151-153.

Scott, O., Williams, G.J. \& Fiddler, G.I. (1980) Results of 24 hour ambulatory monitoring of electrocardiogram in 131 healthy boys aged 10 to 13 years. Br. Heart J., 44, 304-308.

Scoott, C. \& Dalton, R. (2000) Vegetative Disorders.
In: Nelson Textbook of Pediatrics, edited by R.E. Behrman, R.M. Kliegman, Philadelphia, Saunders, pp. 66-104.

Task Force of the European Society of Cardiology and the North American Society of Pacing and Electrophysiology (1996) Heart rate variability: Standards of measurement, physiological interpretation and clinical use. Circulation, 93, 1043-1065.

Wu, H.H., Chen, M.Y., Lee, Y.H., Lin, T.L., Lin, S.N., Chen, K.K., Yin, J.H., Huang, J.K. \& Chang, L.S. (1988) Urodynamic studies in primary nocturnal enuresis. Zhonghua Yi Xue Za Zhi (Taipei), 41, 227-232.

Yakinci, C., Mungen, B., Durmaz, Y., Balbay, D. \& Karabiber, H. (1997) Autonomic nervous system functions in children with nocturnal enuresis. Brain Dev., 19, 485-487.

Yang, T.F., Wong, T.T., Chang, K.P., Kwan, S.Y., Kuo, W.Y., Lee, Y.C. \& Kuo, T.B. (2001) Power spectrum analysis of heart rate variability in children with epilepsy. Childs Nerv. Syst., 17, 602-606. 